\title{
Clustering-Based Threshold Model for Condition Assessment of Concrete Bridge Decks Using Infrared Thermography
}

\author{
Tarek Omar ${ }^{(\bowtie)}$ and Moncef L. Nehdi \\ Department of Civil and Environmental Engineering, Western University, \\ London, ON, Canada \\ \{tomar3,mnehdi\}@uwo.ca
}

\begin{abstract}
Bridge decks are deteriorating at an alarming rate due to corrosion of the reinforcing steel, requiring billions of dollars to repair and replace them. Nowadays, infrared thermography (IRT) diagnostics represent a mature high-technology field that combines achievements in the understanding of heat conduction, material science, and computer data processing. The high interest in the IRT inspection technique is related to its universal character, high testing productivity and in-service safety. However, the analysis of IRT data to evaluate the condition of concrete bridge decks is still rather qualitative, thus preventing the progressive competition of IRT with other inspection techniques. The goal of this research is to understand the relationship between IRT and deck deterioration, and develop a model to determine delamination quantities in concrete bridge decks using a relatively low cost microbolometer infrared camera. Infrared testing was conducted in-situ on a full-scale bridge deck. The thermal IR images were enhanced and stitched using specially developed Matlab codes to create a mosaicked thermogram of the entire bridge deck. Image analysis based on the K-means clustering technique was utilized to segment the mosaic and identify objective thresholds. Hence, a condition map classifying different categories of delamination severity was created and also validated through the results of other techniques obtained on the same bridge. The findings from this study demonstrate that IRT can provide transportation agencies both quantitative and qualitative indications of subsurface delamination defects, thus assisting efficient maintenance and repair decision-making, and focusing limited funding on the most deserving bridge decks.
\end{abstract}

Keywords: Bridge decks $\cdot$ Delamination $\cdot$ Infrared thermography $\cdot$ Image analysis $\cdot$ Mosaic $\cdot$ Clustering $\cdot$ Cost-effective

\section{Introduction}

Existing bridges represent strategic components of infrastructural networks. Concrete bridges experience loss of integrity and changes in resistance that are time variant, due to environmental exposure, various deterioration mechanisms, and excessive mechanical loading. Deterioration processes in concrete bridges are caused by chemical (e.g. alkali-silica reaction, carbonation, corrosion, crystallization, leaching, sulfate and acid 
attack), physical (e.g. freezing-thawing cycles, creep, fatigue, shrinkage, abrasion, erosion), mechanical (e.g. static and/or dynamic loads, construction faults such as those from premature loading during construction), and biological mechanisms (e.g. accumulation of organic matter, living organisms, fungi, and moss) (Penttala 2009). Such degradation mechanisms can compromise the serviceability and structural integrity. They can lead to structural and functional failures, which are catastrophic, both in terms of human life and economic loss, rendering the task of managing this important asset a complex endeavour that attracts growing attention. According to the Canadian infrastructure report card (2016), $26 \%$ of bridges are in fair, poor and very poor condition while as per the United States' 2013 infrastructure report card, an annual investment of $\$ 20.5$ billion would be needed to eliminate the backlog of deficient bridges in the USA by the year 2028.

Different deterioration processes lead to different types of structural defects (e.g. delamination, spalling, cracking, rebar size reduction) or material alterations (e.g. reduced modulus, changed electrical and chemical properties). However, deterioration is commonly initiated by rebar corrosion, followed by cracking, delamination and spalling of concrete (Gucunski and Nazarian 2010). A major challenge associated with inspecting bridge decks is that subsurface delaminations often increase in severity until the damage becomes too severe for cost-effective repair. Subjective or inaccurate condition assessment is the most critical technical barrier to effective management of concrete bridges since visual inspection is the default bridge inspection methodology, whereas its results heavily depend on the expertise and judgment of bridge inspectors. The application of non-destructive testing (NDT) technologies is one of the effective ways to evaluate bridge deterioration. NDT approaches enable the detection of deterioration processes at early stages and can be incorporated into the inspection process to evaluate hidden defects such as reinforcing steel corrosion or crack propagation. The use of simple non-destructive methods, such as chain drag and hammer sounding, are inexpensive methods, typically yielding primarily qualitative and subjective decisions.

Advanced NDT of concrete bridges has its origins in geophysics. A number of techniques introduced exploit various physical phenomena (acoustic, seismic, electric, electromagnetic, and thermal, etc.) to detect and characterize specific deterioration processes or defects. The most commonly used NDT methods in the onsite assessment and evaluation of concrete bridges are the half-cell potential, impact echo, ultrasonic pulse velocity, ground penetrating radar, and infrared thermography (IRT). In general, all these techniques utilize an approach where the objective is to learn about the characteristics of the medium from its response to the applied excitation (Gucunski et al. 2013). IRT is a remote sensing technology that is commonly applicable for detecting subsurface flaws, such as internal cracks and delaminations in concrete bridge components. Thus, IRT does not require direct access to the surface under inspection because images can be captured from distance using appropriate optical lenses. The subsurface anomalies can be detected on the basis of variable concrete properties, such as density, thermal conductivity and specific heat capacity. The resulting heating and cooling behavior is compared with the surrounding sound concrete (Manning and Holt, 1982). The IR images are commonly displayed in a geospatial format to produce GIS thermal IR maps, which rely on the user interpretation, often resulting in undesired subjectivity (Wu et al. 2012). 


\section{Research Objectives and Methodology}

The goal of this study is to develop a model to determine delamination quantities in concrete bridge decks using passive IRT testing. The primary objectives are: (1) understand the relationship between IRT and deck deterioration; (2) produce condition maps that indicate, classify, and quantify delamination defects in bridge decks. The methodology adopted for achieving the objectives consists of: (i) conducting passive IRT testing on a full-scale concrete bridge deck; (ii) producing a thermal thermogram for the entire bridge deck; (iii) applying a clustering technique to determine threshold values; (iv) producing bridge deck condition maps identifying the severity and quantity of the detected delaminated areas; and (v) validating the achieved results.

\section{Infrared Thermography (IRT)}

Heat transfer takes place in three modes: conduction, convection and radiation. The internal properties of a material play important roles in governing its temperature at equilibrium with its surroundings. For instance, heat capacity is defined as the amount of heat needed to raise the temperature of a unit mass of a material by one degree and describes its ability to store heat. Thermal conductivity is the rate at which heat flows through a material. Thermal diffusivity, on the other hand, is the rate at which temperature changes occur in a material. A high thermal diffusivity means that heat transfer through a material will be rapid and the amount of storage will be small. Conversely, low thermal diffusivity indicates a slower rate of heat transfer and a large amount of heat storage (Bagavathiappan et al. 2014). There are two testing approaches for IR thermography based on the source of heat. The active approach uses an external thermal stimulus to induce the required heat flow condition on the concrete under testing. The passive approach uses natural heat sources such as solar heating and ambient temperature changes (Robert 1982).

IRT is a technology to detect thermal radiation emitted from materials. It creates an image of surface temperatures based on the emitted radiation. Thermal IR radiant energy is emitted from all objects that have a temperature greater than absolute zero. The rate at which this energy is emitted is a function of the temperature of the material and its emissivity. A material's emissivity is the ability of its surface to emit energy by radiation relative to a black body and has a value between 0 and 1 . This value is typically greater than 0.9 for concrete. The concept behind the application of IRT in concrete bridge evaluation is that subsurface defects such as delamination disrupt the heat transfer through the concrete. When the temperature of the concrete increases, such as during daytime when the sun and ambient environment are heating the concrete, the surface area above a subsurface delamination warms up at a faster rate than that of surface areas where the concrete is intact. Delaminations can be detected as "hot spots" on the surface of the material, relative to intact concrete. Conversely, during nighttime, the air temperatures usually decrease and the material cools. Thus, the surface area above the delaminations cool at a faster rate than the intact concrete and appear as "cold spots" relative to the intact concrete (Washer et al. 2009). 
ASTM D4788-03, (2013) describes the standard procedure and equipment necessary for conducting passive IR testing for detecting delaminations in bridge decks. Time of data collection is the most critical factor in a passive IR survey. In a study conducted by Washer et al. (2009) on concrete bridges, it was found that the effective time to perform a thermal IR test depends on the depth of the delamination. The most contrast appears on the thermal IR image approximately $4 \mathrm{~h}$ after sunrise for a 2 in. deep delamination and $7 \mathrm{~h}$ after sunrise for a 3 in. deep delamination. Environmental conditions, such as wind speed, solar energy, humidity and ambient temperature also have an influence on the results from a thermal IR bridge inspection (Washer et al. 2009). However, there has been limited application of the IRT technology to inspect full-scale bridges outside the laboratory environment. In the majority of IRT testing, the analysis of IR images depends on the analyzer's personal experience, where the defective areas are identified and calculated based on a visual interpretation approach. Such qualitative data analysis provides quick decisions using the difference in the temperature of the region of interest and a reference region. Although, this procedure is useful in detecting and calculating delaminated and spall areas present in bridge decks, it usually produces inconsistent results.

\section{Field Survey and Data Collection}

Field testing was conducted in this study on an in-service reinforced concrete bridge located in the province of Quebec, Canada and scheduled, by the Ministry of transportation of Quebec for rehabilitation. Delamination, corrosion and high chloride ion content were recently indicated through a condition survey of the bridge. Therefore, the bridge was considered as a good candidate for evaluation study using thermal IR to detect subsurface delamination within the bridge deck. The bridge was constructed in 1969 , and the critical characteristics of the deck include a total length of $147 \mathrm{ft}$ with a transverse width of $29.53 \mathrm{ft}$, which translates into $26.25 \mathrm{ft}$ of drivable surface (supports one lane of traffic in each direction) with one side shoulder. Figure 1 displays the condition of the surveyed deck.

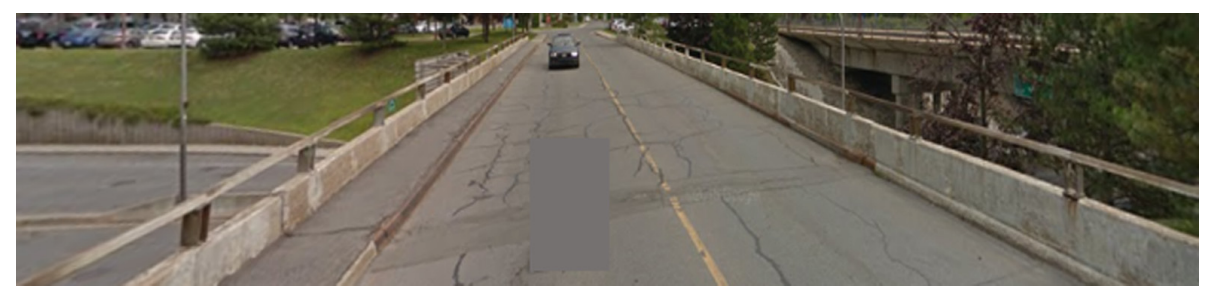

Fig. 1. Photo of the existing condition of the surveyed bridge deck

The most important parameters that must be considered before choosing an IR camera include the spectral range, spatial resolution, temperature range and frame rate. For observing objects at ambient temperature, a long wave length band (7.5-14 $\mu \mathrm{m})$ 
are preferable to detect small thermal contrasts. The spatial resolution of an IR camera primarily depends on the object-to-camera distance, lens system and detector size. An advanced infrared thermal camera, FLIR T650sc, was utilized in this survey. The camera has uncooled microbolometer detector, 5 MP built-in digital camera, and displays thermal images with a resolution of $640 \times 480$ pixels. The thermal IR camera was mounted on a vehicle at a height of $8.5 \mathrm{ft}$ oriented facing straight-down to the concrete deck surface. A calibrated $13.1 \mathrm{~mm}$ focal lens $\left(45^{\circ}\right.$ wide lens $)$ was utilized. This allows a $7 \mathrm{ft} \times 5.3 \mathrm{ft}$ field of view for each image and to survey each single lane with only two passes. The vehicle was driven at low speed over the center of each pass of the bridge deck. The images were taken every $3 \mathrm{ft}$ and hence, a total of 392 thermal and digital images were collected, which covers the entire bridge deck.

The survey was conducted on October $27^{\text {th }}, 2015$ at 1 PM, 6 h after sunrise. During the survey indicated, the ambient temperature was $56^{\circ} \mathrm{F}$, relative humidity was $26 \%$, wind speed was $5 \mathrm{mph}$. Bridge deck dryness was considered during data collection since surface moisture can reduce the thermal contrast on the thermal IR images. Sun direction was also considered to avoid shadows on images. The total time of data collection was about two hours. The digital images were used to separate patches and surface defects from subsurface anomalies. The Matlab image acquisition toolbox was utilized to initialize acquisition and storage of the IR data. Figure 2 illustrates the utilized camera and the vehicle mounted setup.

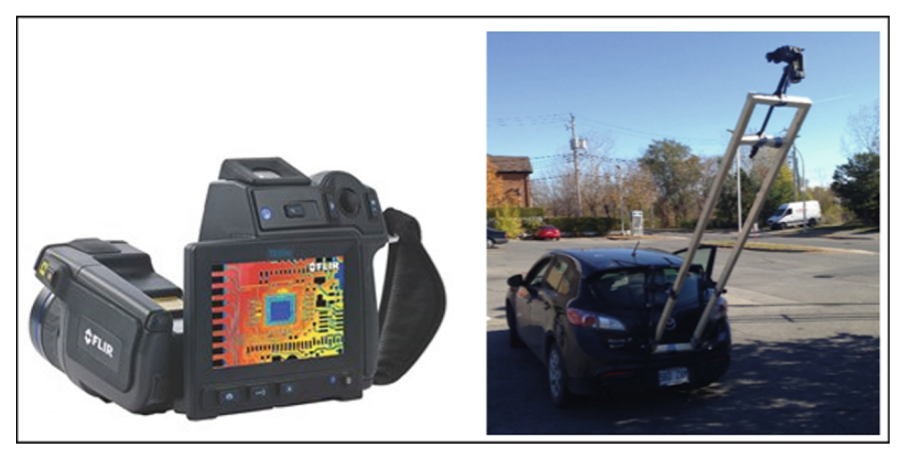

Fig. 2. Illustration of FLIR T650sc IR camera and vehicle mounted setup system

\section{Clustering-Based Threshold Model}

The developed model to extract quantitative information, such as size and location of defective areas from the captured IR images consists of several procedures. At first, the captured IR images of the bridge deck were enhanced using several functions in the FLIR Tools+ and ResearchIR software to improve the images' quality. For instance, the Gaussian smoothing filter was used to reduce noise whereby the software selects the size of the filter automatically. The histogram equalization function was used to distribute the intensities on the histogram, which in turn enhanced the images' thermal contrasts. The thermal MSX function (multi-spectral dynamic imaging), which is a 
fusion algorithm that embosses visible spectrum details (from the digital camera) onto the infrared image, was used to enable sharper-looking images and quicker target orientation. This pre-processing was required for further analysis and to differentiate delaminated areas from surface features (discoloration, oil stains, sand and rust deposits, etc.) that appear in the infrared image, yet are unrelated to subsurface conditions. The temperature data of each image was then saved in a separate Excel file using the ResearchIR software.

Figure 3 illustrates the algorithm developed in the present study to create a mosaicked thermogram of the entire bridge deck and to analyse the IR data. A selected window from each image was extracted and stitched together with the extracted window from the next image and so on. The height of each stitch window was the number of pixels that are equivalent to the images' spacing. Hence, this appending process produced continuous data for each survey pass. The dimension in pixels depends upon the pixel resolution in each direction. For the surveyed bridge, the dimension of the stitched strip was $600 \times 13,230$ pixels and $2400 \times 13,230=31.75$ million pixels for the entire two lane deck. A Matlab code was written by the authors to extract and stitch the selected pixels from each image. For visualization, it was necessary to scale-down the data dimension of the mosaic. This was achieved by assuming

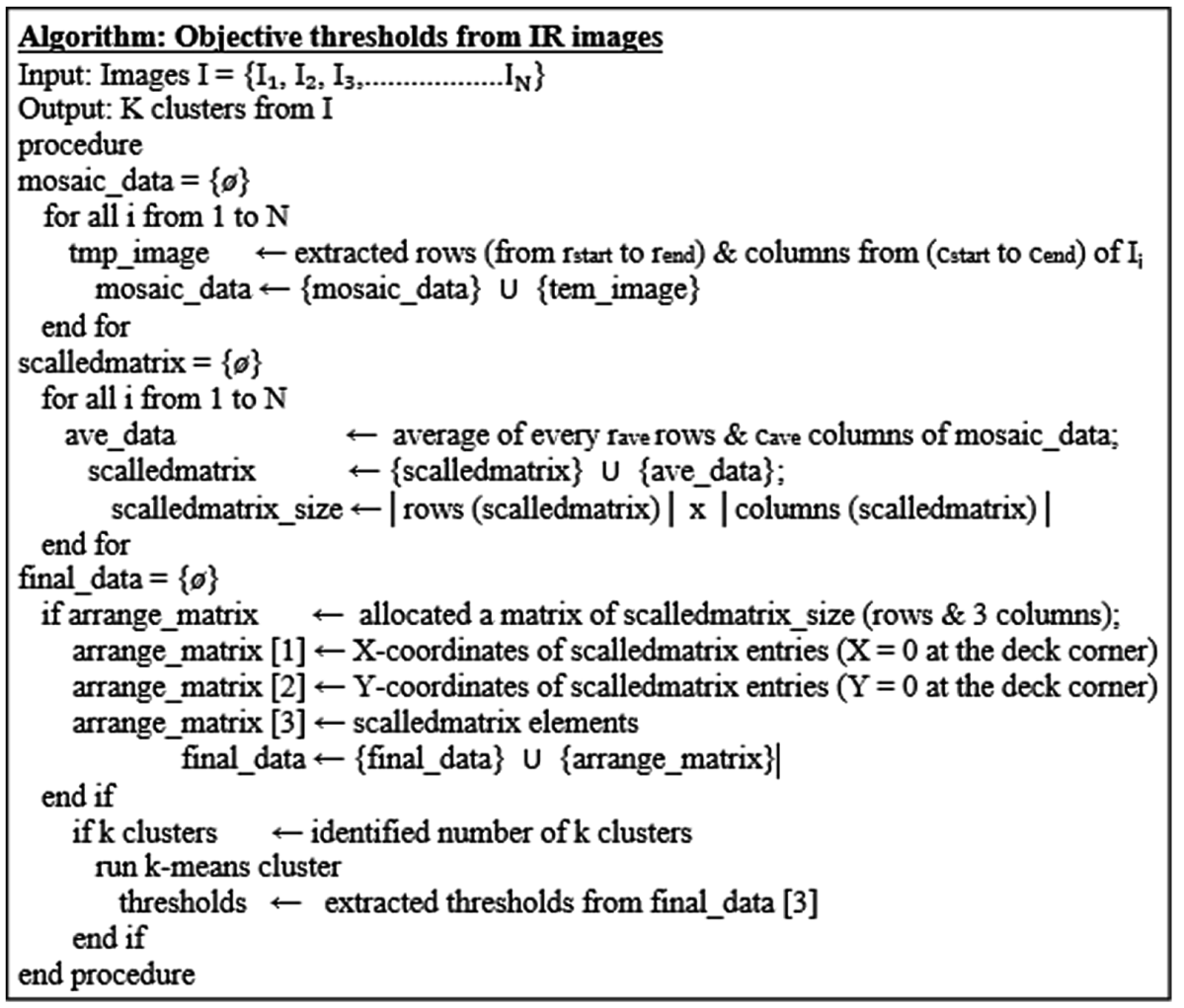

Fig. 3. Proposed algorithm for thresholds from IR images 
that the entire deck was divided into small $10 \times 10 \mathrm{~cm}$ area boxes, with temperature unable to change within each box, then, computing the average of pixels, which represents the resolution within the selected dimension. This resulted in a reduced file with $80 \times 450=36,000$ pixels. The mean function in Matlab was modified and a new code to calculate the mean for specified dimension of input data was written. Another Matlab code was also written by the authors to identify the co-ordinates of each pixel in the temperature data file. The origin point $(0,0)$ was assigned to the corner of the bridge deck where the survey started and then to all other pixels in the file. The final analysis output was an Excel file including pixel information (co-ordinates and temperature).

Clustering analysis is an unsupervised technique defined as a statistical classification method for discovering whether the individuals of a population fall into different groups by making quantitative comparisons of multiple characteristics (Melhem and Cheng 2003). The K-means clustering is the most commonly used method for statistical data analysis because of its ease of implementation, efficiency and empirical clustering. In addition, this clustering algorithm can identify important features in the data to be classified, characterize the degree of similarity and organize the data through cluster prototypes (Jain 2010). The K-means procedure divides the N-dimensional population into $\mathrm{K}$ sets of clusters. The algorithm arbitrarily specifies an initial mean vector (seed) for each of the K clusters. Each data point is then assigned to a class such that the squared error between the empirical mean of a cluster and the points in the cluster is minimized. A new set of cluster mean vectors is then calculated from this classification and the data are rearranged. The iterations are continued until there is no significant change in data points' assignments from one iteration to the next or a maximum number of iterations is achieved (Jain 2010).

Thresholding classification using the K-means clustering was utilized in the present study to overcome subjective selection of threshold values. The number of clusters (k) is the user defined parameter to perform k-means clustering for a data set. To classify the IRT data, the number of condition categories (clusters) was identified based on a framework developed by the authors as illustrated in Fig. 4. The framework is based on the age of the bridge deck, the thermal contrast values obtained from the IR images, and known criteria of deterioration, which can be determined by visual inspection of the deck. For instance, it is unlikely that deterioration initiates in bridge decks under ten years of age. ASTM D4788-03 of passive IRT testing indicates that a temperature difference between the delaminated or de-bonded area and the adjacent solid concrete of at least $0.5^{\circ} \mathrm{C}$ must exist to identify delamination by an imaging infrared scanner. The Ontario Structure Inspection Manual (OSIM, 2008) differentiates between light, medium and severe spalling signs based on physical measures on the bridge deck. Spalling was chosen because it is mainly the continuation of the delamination process and can be physically measured. 


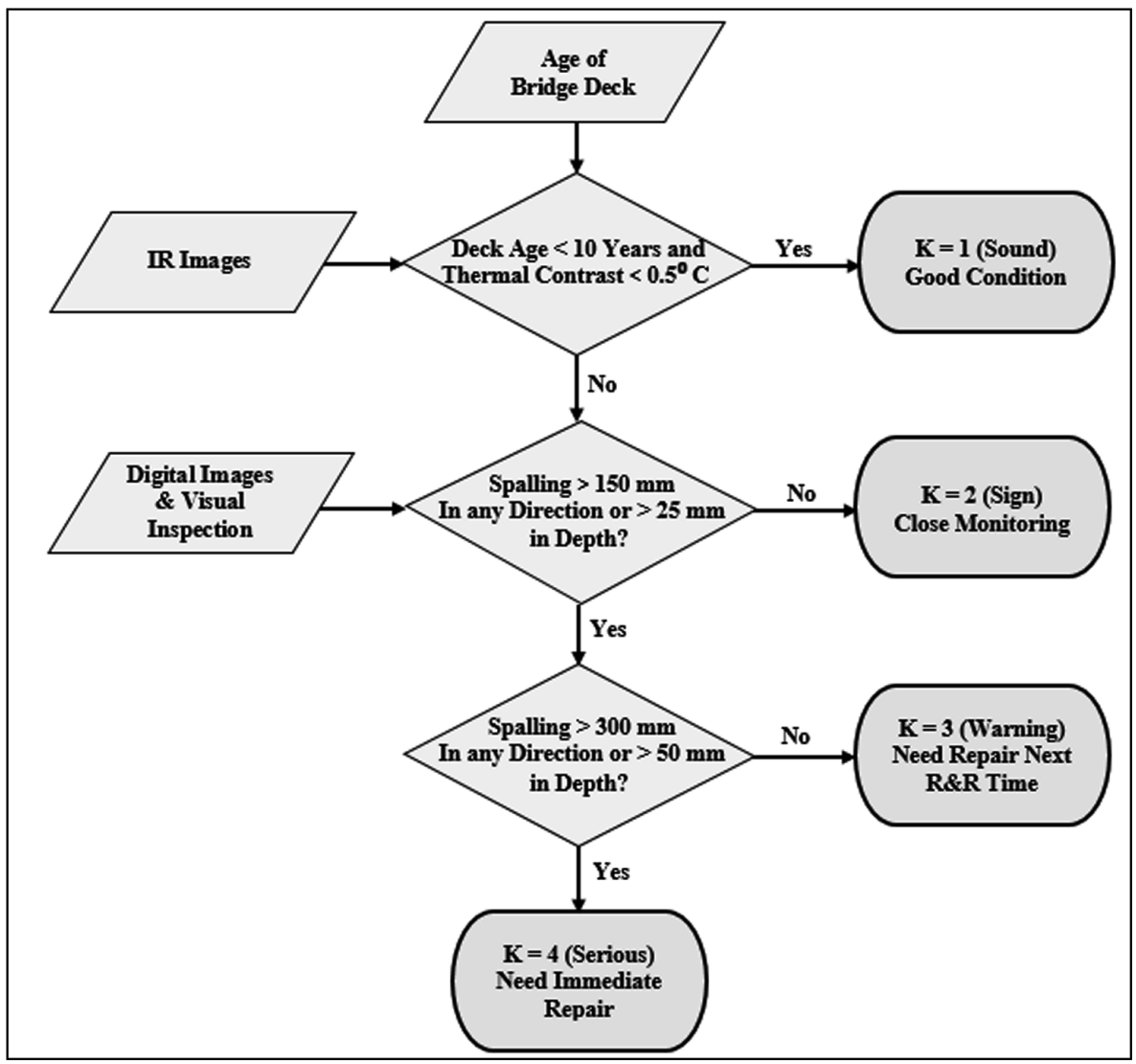

Fig. 4. Flowchart for determining number of condition categories (K)

\section{IRT Results and Discussion}

The bridge deck being considered is about 47 years old. Its recorded thermal contrast was higher than $0.5^{\circ} \mathrm{C}$, and maximum spalling of $225 \mathrm{~mm}$ was measured. Hence, three clusters were determined to differentiate between the sound and delaminated concrete regions. The K-means clustering algorithm in Matlab was applied to identify the thresholds. Based on the identified thresholds, the temperature values were grouped into the same number of clusters and uploaded in a commercial mapping software to construct a high contrast composite condition map accurately delineating the severity of subsurface delaminations as illustrated in Fig. 5. Sound concrete areas were represented by a green color, concrete areas that require close monitoring were represented by a yellow color, and warning concrete areas that require repair were represented by a red color. The temperature differential between the sound and unsound areas of the concrete deck was about $8^{\circ} \mathrm{C}$ where the sound concrete areas had the lowest temperature values and the warning concrete areas had the highest temperature values. The 


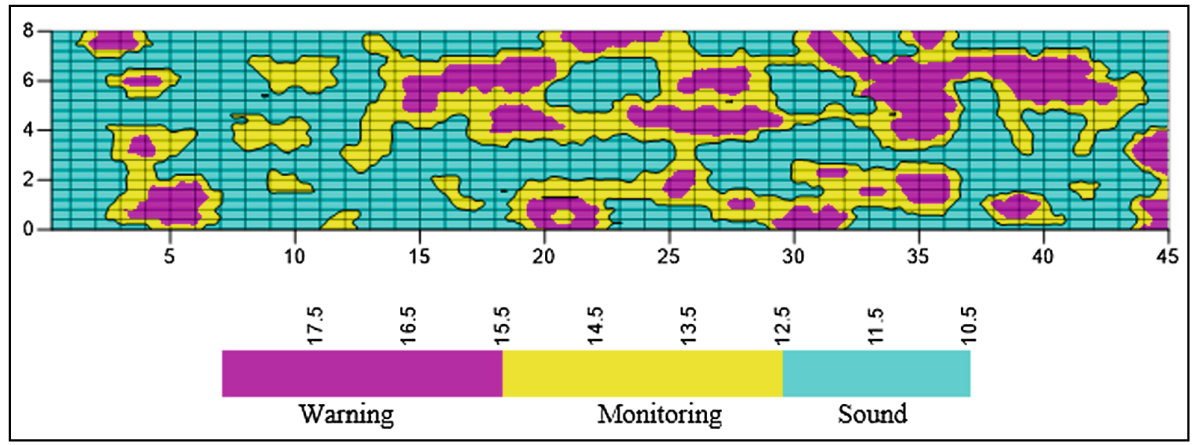

Fig. 5. IRT condition map of the surveyed bridge deck indicating severity of delamination

reason for this is that, during the day, a warming trend existed such that the targets had positive thermal contrasts, which meant that the delaminated regions were at a temperature higher than that of the sound concrete. This is the anticipated behavior based on the fundamental heat transfer theories and complies with previous results reported by Washer et al. (2013) and Chase et al. (2015).

Creating such accurate condition map with highlighted delamination categories from thermal IRT data is useful to the bridge management team and bridge inspectors responsible for ratings. Producing a plan view mosaic of the entire bridge deck in the presented study facilitated the application of thresholding classification to identify and categorize subsurface delaminated areas. The thresholds between the three condition categories were determined based on the developed clustering process without analyst interference and, thus providing objective classification. The thermal contrast developed from the concrete subsurface provided numerical values for the color contrasts that were used to process data more effectively than simply comparing multiple images. This procedure is useful in detecting and calculating delaminated areas present in the bridge deck. In addition, calculating the total delaminated areas in different categories provides a quantified basis for powerful decision making and hence, can be paramount in prioritizing alternatives for bridge deck repair. The percentage of delaminated areas in each condition category was calculated by computing the total pixels in the same category over the total pixels in the entire deck area and summarized in Table 1.

Table 1. Percentage of defected areas in the surveyed bridge

\begin{tabular}{l|l|l|l|l|l}
\hline Bridge element & NDT survey type & Survey result & \multicolumn{3}{|l|}{ Concrete condition } \\
\cline { 4 - 6 } & & & Sound & Monitoring & Warning \\
\hline \multirow{2}{*}{ Bridge deck } & IRT & Present Study & $58 \%$ & $17 \%$ & $25 \%$ \\
\cline { 2 - 6 } & GPR & MOT Record & $62 \%$ & $16 \%$ & $22 \%$ \\
\cline { 2 - 6 } & Hammer sounding & MOT Record & $40 \%$ & & \\
\hline
\end{tabular}




\section{Validation with Other Techniques}

The bridge investigated by IRT in the present study was previously surveyed using GPR scanning in September, 2015 by Radex Detection Inc., a consulting firm specializing in concrete structure scanning, as part of a condition survey program. GPR evaluates the condition of concrete bridge decks based on the difference between reflection amplitudes of the top rebar layer, where strong reflection indicates sound concrete, while the area with high amplitude attenuation is commonly associated with the corrosion of steel reinforcement. The scan was conducted using GSSI-SIR3000 with $1600-\mathrm{MHz}$ antenna frequency. Data collection was made longitudinally on the deck where the spacing between the GPR profiles was $500 \mathrm{~mm}$ and, hence each GPR profile represented a zone width of $500 \mathrm{~mm}$. Visual interpretation of GPR data refers to those techniques that are based on visual recognition of deterioration in a GPR data set (Tarussov 2013). The GPR profiles of the surveyed bridge were analyzed by Radex based on a line scan (B-scan) visual image analysis technique. The analyst scrolls through each GPR profile and marks visible anomalies based on known criteria of deterioration. The processed profiles are then combined by a proprietary software to create a corrosion map delineating the corrosion severity as illustrated in Fig. 6. In addition, three core drilling concrete samples for visual and chloride ion concentration analysis were taken from the bridge deck where two cores were taken at corrosion areas and one core was taken at a sound concrete area. For the two cores located at corrosion areas, the chloride ion concentrations were detrimental to the concrete when they were $0.04 \%$ by concrete mass, while the commonly accepted chloride threshold that facilitates rebar corrosion is $0.025 \%$. This result validates the corrosion identified by the analysis of the GPR scan data.

In spite of the different mechanisms of both IRT and GPR techniques and their capabilities of detecting different deterioration types, the IRT delamination map achieved by the developed analysis in Fig. 5 was compared with the corrosion map provided by the GPR scanning in Fig. 6. Though the shapes (geometry) of the two analyses do not match exactly, the areas in which delaminations/corrosion were detected are mostly the same. The percentages of the corroded areas in each category were computed and are summarized in Table 1. For further validation, the location and

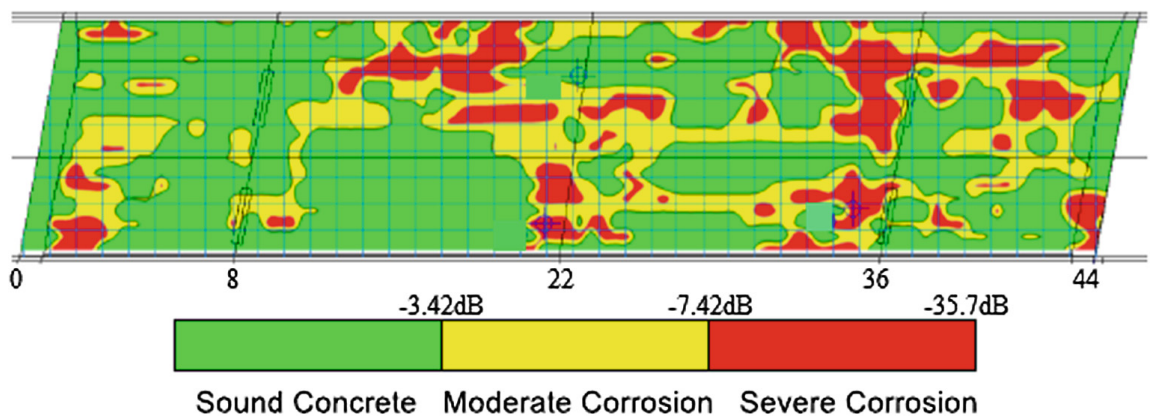

Fig. 6. GPR corrosion map of the surveyed bridge as per the record of MOT, Quebec 
geometry of subsurface defected regions were identified with considerably similar results using hammer sounding, which was conducted by the inspection team from the ministry of transportation of Quebec. The percentage of delaminated areas identified by the hummer sounding test were computed and are presented in Table 1.

The GPR results showed $38 \%$ total corroded areas on the bridge deck, whereas the total delaminated area calculated from the IRT results was $42 \%$ (about $10 \%$ difference in total defects between the IRT and GPR surveys). This complies with the results of previous studies. For instance, Maser et al. (2009) reported that in most bridge structures, areas of severe corrosion are associated with delamination even if cracks are not detected directly. Washer et al. (2013) reported that not all delamination can be observed in GPR images and both GPR and IRT can be used together for accurate identification of anomalies in highway bridges. The hammer sounding results showed $40 \%$ total delaminated areas on the bridge deck (40\% of the total delaminations is common between these two surveys). Thus, the IRT analysis procedure developed in the present study identified the delaminated areas with a superior degree of agreement with both GPR scan and hummer sounding.

\section{Concluding Remarks}

IRT has several advantages over other NDT techniques, such as being able to scan a large area in a non-contact manner. It is much faster than conventional methods and produces visual thermal images immediately in the field. Moreover, IRT has a potential to be applied at near highway speed over a bridge deck, which can help reduce traffic disruption and conduct bridge inspections at a faster rate. In addition, the IRT testing can be conducted during both day and night time. Conversely, IRT is dependent upon environmental conditions and can become ineffective if such conditions are unfavorable. A robust analysis procedure of IRT data was developed herein to detect and classify the severity of subsurface defects in concrete bridge decks. The findings are presented in terms of condition maps delineating the severity of delamination. The boundaries between the condition categories were determined based on the threshold clustering technique applied on the recorded concrete surface temperature, thus providing objective threshold values and accurate classification. Accordingly, the percentage of delaminated areas in the bridge decks were calculated with reasonable accuracy. Once the thermal IR images become available, the developed procedure can analyze and determine whether a defect exists with minimum human interference. The subsurface defected regions identified by the developed analysis provided satisfactory and acceptable results and were confirmed by other NDT techniques including GPR scans and hummer sounding tests. 


\section{References}

ASTM Standard D4788-03: Standard Test Method for Detecting Delaminations in Bridge Decks Using Infrared Thermography. ASTM International, West Conshohocken (2013). www.astm. org

Bagavathiappan, B., Lahiri, T., Philip, J., Jayakumar, T.: Infrared thermography for condition monitoring - a review. J. Infrared Phys. Technol. 60, 35-55 (2014)

Chase, S., Adu-Gyamfi, Y., Tunuguntla, P.: Bridge deck sub-surface defect detection using time-lapse thermography. In: 94th Annual Meeting, pp. 1-22 TRB, Washington, D.C. (2015)

Gucunski, N., Imani, A., Romero, F., Nazarian, S., Yuan, D., Wiggenhauser, H., Shokouhi, P., Taffe, A., Kutrubes, D.: Non-destructive testing to identify concrete bridge deck deterioration. In: 92nd Annual Meeting, 96 p., TRB, Washington, D.C. SHRP 2 Report (2013)

Gucunski, N., Nazarian, S.: Material characterization and condition assessment of reinforced concrete bridge decks by complementary NDE technologies. In: Proceeding of Structures Congress, Orlando, Florida, United States, pp. $429-439$ (2010)

Jain, K.: Data clustering: 50 years beyond K-means. J. Pattern Recogn. Lett. 31(8), 651-666 (2010)

Melhem, H., Cheng, Y.: Prediction of remaining service life of bridge decks using machine learning. J. Comput. Civ. Eng. 17(1), 1-9 (2003)

Manning, D., Holt, F.: Detecting delaminations in concrete bridge decks. J. Concr. Int. 34, 1-8 (1982)

Maser, K.: Integration of ground penetrating radar and infrared thermography for bridge deck condition testing. J. Mater. Eval. 66(11), 1130-1136 (2009)

Penttala, V.: Causes and Mechanisms of Deterioration in Reinforced Concrete, 323 p. Woodhead Publishing Limited, CRC Press, Sawston (2009)

Robert, M.: Science behind thermography-thermal infrared sensing for diagnostics and control. J. Thermosense 371, 2-9 (1982)

Tarussov, A., Vandry, M., De La Haza, A.: Condition assessment of concrete structures using a new analysis method: ground-penetrating radar computer-assisted visual interpretation. J. Constr. Build. Mater. 38, 1246-1254 (2013)

Washer, G., Fenwick, R., Nelson, S.: Guidelines for the thermographic inspection of concrete bridge components in shaded conditions. In: 92nd Annual Meeting, TRB, Washington, D.C. pp. 1-14 (2013)

Washer, G., Fenwick, R., Harper, J.: Effects of environmental variables on infrared imaging of subsurface features in concrete bridges. J. Transp. Res. Board 2108(2109), 107-114 (2009)

$\mathrm{Wu}, \mathrm{X}$., Yao, H., Xie, D., Xu, Z.: Developing of management information system of road and bridge infrastructure based on ArcGIS engine. In: 2nd International Conference, Remote Sensing Environment and Transportation Engineering (RSETE), pp. 1-3. IEEE (2012) 